\title{
Linx
}

Revue des linguistes de l'université Paris X Nanterre

$59 \mid 2008$

Les conjonctions en diachronie : parcours sémantiques

\section{Alice Krieg-Planque (2009). La notion de " formule » en analyse du discours. Cadre théorique et méthodologique}

Besançon, Presses Universitaires de Franche-Comté, 145 pages

\section{Fanny Rinck}

\section{OpenEdition}

\section{Journals}

Édition électronique

URL : http://journals.openedition.org/linx/650

DOI : 10.4000/linx.650

ISSN : 2118-9692

\section{Éditeur}

Presses universitaires de Paris Nanterre

\section{Édition imprimée}

Date de publication : 1 décembre 2008

Pagination : 187-189

ISSN : 0246-8743

\section{Référence électronique}

Fanny Rinck, «Alice Krieg-Planque (2009). La notion de «formule » en analyse du discours. Cadre

théorique et méthodologique », Linx [En ligne], 59 | 2008, mis en ligne le 08 juillet 2011, consulté le 21 septembre 2020. URL : http://journals.openedition.org/linx/650 ; DOI : https://doi.org/10.4000/linx. 650

Ce document a été généré automatiquement le 21 septembre 2020

Département de Sciences du langage, Université Paris Ouest 


\section{Alice Krieg-Planque (2009). La notion de " formule » en analyse du discours. Cadre théorique et méthodologique}

Besançon, Presses Universitaires de Franche-Comté, 145 pages

\section{Fanny Rinck}

\section{RÉFÉRENCE}

Alice Krieg-Planque (2009). La notion de "formule » en analyse du discours. Cadre théorique et méthodologique. Besançon, Presses Universitaires de Franche-Comté, 145 pages.

1 "Développement durable ", "purification ethnique ", "fracture sociale ", "principe de précaution", autant de formules incontournables dans les débats publics, sans cesse reprises et commentées, qui s'imposent à nous comme des évidences aussi bien qu'elles questionnent : mais que signifient-elles exactement?

2 C'est sur ce type de formules qu'Alice Krieg-Planque développe depuis plusieurs années ses recherches en analyse de discours, et c'est sur la notion même de "formule » que porte l'ouvrage dont nous rendons compte ici. Cet ouvrage fait suite à la thèse d'A. Krieg-Planque (2000) $)^{1}$ et à la parution d'une version remaniée $(2003)^{2}$ qu'il restait selon l'auteure à compléter par ce qu'elle a conçu comme un "précis » sur la notion de formule en analyse de discours ; à travers un opus concis, il s'agit d'expliciter l'arrièreplan théorique de la notion et de fournir un cadre méthodologique pour les analyses de corpus.

3 La notion de formule désigne « un ensemble de formulations qui, du fait de leurs emplois à un moment donné et dans un espace public donné, cristallisent des enjeux politiques et sociaux que ces expressions contribuent dans le même temps à construire» (p.7). Cette notion se rattache aux études sur les usages sociaux et politiques du lexique, en tant qu'il est porteur de valeurs, d'arguments, d'engagements et se trouve au cœur du débat public (chapitre 1). Elle trouve ses fondements 
conceptuels dans les travaux de J.-P. Faye (chapitre 2) et de M. Ebel et P. Fiala (chapitre 3). Le chapitre 4, central, permet d'en spécifier les propriétés.

4 L'auteure souligne que seule une analyse fine permet d'évaluer en quoi une séquence formelle présente un caractère formulaire. La formule est une catégorie graduelle: " une séquence est plus ou moins formule selon qu'elle remplit plus ou moins chacune des quatre propriétés caractéristiques » identifiées par l'auteure.

5 La première propriété d'une formule est d'être une séquence formelle relative-ment figée, ce qui va de pair avec sa seconde propriété, d'ordre discursif : sa circulation dans l'espace public et sa notoriété. Ainsi les candidats à la formule sont-ils des segments bien identifiés, qui peuvent se limiter à un morphème («crise ») mais se présentent le plus souvent sous une forme relationnelle: des noms composés (comme «sans-papiers »), des syntagmes de type nom - préposition - nom (comme "devoir de mémoire »), et, de manière privilégiée, des syntagmes à adjectifs dénominaux («fracture sociale »), et des noms présupposant, au niveau sémantique, l'existence d'un verbe attesté ("intégration », "mondialisation", "démocratisation») ou non ("copinisation»). La polysémie des unités lexicales et les ambiguïtés quant à leurs relations favorisent les conflits d'interprétation.

6 La productivité lexicologique, les défigements et les détournements sont autant d'indices du caractère notoire de la formule, qui se doit non seulement d'être fréquente à un moment donné mais également d'être attestée dans des discours variés, ordinaires et savants, oraux et écrits. La formule fonctionne discursivement comme un passage obligé, qui sert de cadrage aux débats publics et en cela aussi de contrainte par rapport à laquelle chacun doit se situer.

Une formule a en effet pour troisième propriété d'être un référent social, signe connu de tous et qui évoque quelque chose pour tous, comme le montrent ses emplois sur le mode du présupposé (en particulier dans les titres de presse, comme dans «banlieues: le constat d'échec »).

8 La valeur de description des faits politiques et sociaux portée par la formule en fait l'objet de polémiques, et c'est sa quatrième propriété. Chargée d'enjeux, la formule est au cœur des débats et suscite en elle-même le débat : on observe des refus de l'énoncer, des injonctions à l'utiliser, des propositions de mots concurrents, des commentaires sur son (in)adéquation à ce qu'elle désigne, ou même sur la réalité ou l'inconsistance de ce qu'elle est supposée désigner, etc. Les polémiques se manifestent à travers des opérations métadiscursives qui accompagnent les usages de la formule et permettent d'étudier ce que l'auteur appelle les achoppements des locuteurs sur ces segments, et à travers eux les enjeux socio-politiques qu'ils recèlent.

9 Dans le dernier chapitre, l'interdisciplinarité est à l'honneur : après une caractérisation discursive de la notion de formule, il s'agit pour l'auteure de mobiliser les sciences de l'information et de la communication pour interroger la notion d'espace public et le rôle des médias dans la circulation des formules. Elle suggère que ce ne sont pas tant les médias que les acteurs sociaux qui sont à l'origine des formules et que les médias sont des agents de diffusion qui contribuent à leur acceptabilité.

La notion de formule a vocation à être une notion clé en analyse du - et des - discours. Elle donne une assise matérielle à une réflexion critique sur les discours dominants, qu'ils soient d'ordre politique, médiatique ou institutionnel. Gageons que le précis décrit ici deviendra un ouvrage de référence pour les études portant sur des corpus 
issus de l'un de ces champs et pour les études les abordant conjointement, à travers la circulation des discours.

11 Ces études devraient permettre de creuser plus avant le pouvoir d'action de ces énoncés «magiques» que sont les formules 1) en étudiant ce qui en est dit dans les discours et comment elles sont mobilisées dans les situations d'analyse des faits sociopolitiques et de prise de décision; 2) en rapprochant la notion, après l'en avoir distinguée, d'autres notions clés pour l'étude des discours et de l'argumentation, en particulier les topoï et les préconstruits culturels, cités par l'auteure (p. 69), mais peutêtre aussi la notion de catégorisation en psychologie sociale et celle de schématisation chez J.-B. Grize ; 3) en poursuivant les collaborations interdisciplinaires sur les questions soulevées par l'ouvrage: qu'en est-il des enjeux des formules en termes de mémoire, de leurs « effets sur les croyances et les consciences» (p. 104), des « rapports de pouvoir et d'opinion» (p. 30) qui se structurent et s'observent dans le discours ? C'est en cela en somme que la notion de formule nous semble particulièrement heuristique : elle invite à approfondir le lien entre discours, cognition et culture au prisme de l'interdiscours.

\section{AUTEURS}

\section{FANNY RINCK}

Modyco, Université Paris Ouest Nanterre La Défense 